\title{
Active penetration of Trypanosoma cruzi into host cells: historical considerations and current concepts
}

\author{
Wanderley de Souza $^{1,2}$ and Tecia M. Ulisses de Carvalho ${ }^{1 *}$ \\ ' Laboratório de Ultraestrutura Celular Hertha Meyer, Instituto de Biofísica Carlos Chagas Filho, Universidade Federal do Rio de Janeiro, Rio de Janeiro, Brazil \\ ${ }^{2}$ Instituto Nacional de Metrologia, Qualidade e Tecnologia, Rio de Janeiro, Brazil
}

\section{Edited by:}

Ricardo T. Gazzinelli, University of Massachusetts Medical School, USA

Reviewed by:

Lynn Soong, The University of Texas Medical Branch, USA

Emilio L. Malchiodi, University of

Buenos Aires, Argentina

\section{*Correspondence:}

Tecia M. Ulisses de Carvalho,

Laboratório de Ultraestrutura Celular Hertha Meyer, Instituto de Biofísica

Carlos Chagas Filho, Universidade

Federal do Rio de Janeiro, CCS-Bloco

$G$, Ilha do Fundão, 21941-900

Rio de Janeiro, Brazil.

e-mail: tecia@biof.ufrj.br
In the present short review, we analyze past experiments that addressed the interactions of intracellular pathogenic protozoa (Trypanosoma cruzi, Toxoplasma gondii, and Plasmodium) with host cells and the initial use of the term active penetration to indicate that a protozoan "crossed the host cell membrane, penetrating into the cytoplasm." However, the subsequent use of transmission electron microscopy showed that, for all of the protozoans and cell types examined, endocytosis, classically defined as involving the formation of a membrane-bound vacuole, took place during the interaction process. As a consequence, the recently penetrated parasites are always within a vacuole, designated the parasitophorous vacuole (PV).

Keywords: Trypanosoma cruzi, endocytosis, active penetration, phagocytosis, Chagas disease

\section{INTRODUCTION AND EARLY STUDIES}

Investigation of the interactions of Trypanosoma cruzi with host cells became possible after techniques to cultivate this protozoan in tissue culture were developed. The first approach was described by Kofoid et al. (1935), who showed that the protozoan could survive and multiply in cultures of heart cells from mouse and rat embryos. A subsequent study by Romaña and Meyer (1942) using chick embryo heart cell cultures described the behavior of T. cruzi in tissue cultures in detail. Theirs in vitro observations of the interaction process in both living and fixed cultures led the authors to describe two mechanisms of cell infection by the protozoan: active penetration and phagocytosis. In this classic paper, it was stated that "in general, the active penetration was more visible with metacyclic forms that, with their great motility, easily crossed the cell surface, penetrating into the protoplasm of fibroblasts and myocytes." To the best of our knowledge, this is the first reference to the active penetration of parasitic protozoans into cells. Six years later, Meyer and Xavier de Oliveira (1948) published a paper and a small book confirming the initial observations and reported that the parasite "can touch the surface of the host cells without penetration. Occasionally, they adhere to the cell surface and suddenly penetrate into the cell." These observations were more clearly demonstrated in a classic film by H. Meyer and A. Barasa. Part of that film which is kept in our laboratory (founded in 1940 by Hertha Meyer) is available at the site of the Brazilian Society of Protozoology (www.sbpz.org.br). The same basic idea initially proposed by Meyer and colleagues was presented in 1973 by Dvorak and Hyde (Dvorak and Hyde, 1973; Hyde and Dvorak, 1973) based on observations of the interaction of T. cruzi trypomastigotes of the Ernestina strain with secondary bovine embryo skeletal muscle cells (BESM) and HeLa cells obtained through phase contrast microscopy under controlled conditions. The process of cell invasion was described as an active penetration process in which mechanical activity of the protozoan is prevalent, and the parasites penetrate into the cell through the plasma membrane. According to these studies, the parasites enter cells posterior end first.

It is important to note, although it is not the focus of the present review, that the same basic idea of active penetration has also been applied to other protozoans, such as Toxoplasma gondii and Plasmodium, which were again analyzed first by Nery Guimarães and Meyer (1942) and Dvorak et al. (1975).

\section{SECOND PHASE}

One of the authors of this review (WS) joined the Hertha Meyer's laboratory in 1969 and began the first studies addressing the interaction of T. cruzi with chick embryo heart muscle cells and macrophages using electron microscopy. Jim Dvorak visited the laboratory several times between 1972 and 1980, and intense discussions about the concept of active penetration took place at that time. With enthusiasm, Jim defended the idea that T. cruzi and Plasmodium are able to generate a transient tunnel-like structure in the host cell plasma membrane that is sealed immediately after parasite internalization, and the parasite then establishes intimate contact with the host cell cytoplasm. Up to this point, electron microscopy had not been used to analyze the parasitic protozoan-host cell interaction process. However, a few images obtained in our laboratory showed that recently penetrated trypomastigotes were not in contact with the myofibers of heart muscle cells but were instead located within a membrane-bound vacuole. These observations were made approximately in 1972 
when Nadia Nogueira was being trained to work with T. cruzi in Hertha Meyer's lab. Subsequently, she moved to Rockefeller University in New York, where she started a project in Zanvil Cohn's laboratory analyzing the interaction of T. cruzi with several different cell types, including mouse peritoneal macrophages, $\mathrm{HeLa}$ cells, L cells, and calf embryo fibroblasts. During a visit to Dr. Cohn's lab, one of the authors of the present paper (WS) had the opportunity to discuss these electron microscopy images with these investigators, which were subsequently published in a classic paper in 1976 (Nogueira and Cohn, 1976). The images showed clearly that all internalized parasites were initially located in a vacuole, designated the parasitophorous vacuole (PV) following the suggestion of William Trager, who described a vacuole akin to that in Nogueira and Cohn's images in erythrocytes infected by Plasmodium (Langreth and Trager, 1973). Similar observations were made in T. gondii by Jones and Hirsch (1972). Since that time, it has become clear that in all situations examined to date, T. cruzi and certain other intracellular protozoan always penetrate the host cell in a process that is better characterized as an endocytic process, involving the initial formation of aPV. This process takes place independent of the nature of the host cell. Even so-called non-professional phagocytic cells can be penetrated by $T$. cruzi and T. gondii. The term induced endocytosis or even induced phagocytosis has been used to refer to a process in which the parasite is able to stimulate endocytic activity in the future host cell.

According to Nogueira and Cohn in the case of T. cruzi, subsequent to penetration, the parasites leave the $\mathrm{PV}$ in a process they described as "escaping" and then enter into direct contact with the host cell cytoplasm. However, a few years later, it was shown (Carvalho and de Souza, 1989) that there is no escaping, but rather, fragmentation of the PV membrane occurs, most likely due to the activity of enzymes secreted by the parasite, as was subsequently shown by Andrews et al. (1990), associated with the complete disappearance of the PV. Taken together, these observations clearly indicate that an endocytic process is always involved during the process of T. cruzi internalization into a host cell. The same basic idea can be extended to other intracellular parasitic protozoa.

\section{A NEW CONCEPT OF "ACTIVE PENETRATION"}

We had the opportunity to discuss the data described above as well as the results obtained by several groups in Plasmodium and Toxoplasma with Hertha Meyer, James Dvorak, Nadia Nogueira, and Zanvil Cohn, and we reached the conclusion that active penetration, as initially defined, does not exist during the process of parasitic protozoa interacting with host cells. A possible exception is observed during infection of host cells by microsporidians, which include a large number of species initially considered to be protozoans but that have more recently been considered to be fungi based on the presence of a large number of genes that, upon phylogenetic analysis, cluster Microsporida with Fungi (see review by $\mathrm{Xu}$ and Weiss, 2005). These organisms present a complex life cycle, and their spores contain a structure known as the polar tube. When one of these organisms attaches to the surface of a host cell, signals activate a process that leads to evagination of a structure known as the polar tube, which then penetrates into the host cell, establishing a type of bridge between the cytoplasm of the spore and the cytoplasm of the host cell. The sporoplasm, which may contain one or two nuclei, then flows through the connecting tube and is transferred to the host cell cytoplasm, where it will develop.

After some discussion, it was concluded that the term active penetration could be used to indicate the fact that the parasite plays an important role in the "induction of the host cell invasion process." Indeed, since the first description of the process, it has been clear that the intense movement of the protozoan, especially due to the flagellar beating process, plays some role. This idea was further analyzed by Schenkman et al. (1991), who showed that maintenance of an active energetic metabolism is fundamental for T. cruzi to invade cells, as this process is prevented by treatment of the parasites with 2-deoxy-glucose, an inhibitor of glycolysis, as well as sodium azide, antimycin, and oligomycin, which interfere with the mitochondrial metabolism involved in the synthesis of ATP.

The term active penetration has also been employed from another perspective. Kipnis et al. (1979), for example, used it to describe the penetration of bloodstream trypomastigotes into macrophages in a process that was only partially inhibited by cytochalasin B. At present, we know that this compound does not inhibit all forms of endocytosis.

Therefore, we can conclude that the available data, especially those obtained through transmission electron microscopy of thin sections, clearly show that recently penetrated T. cruzi of both infective and non-infective forms are always located within a PV that interacts with the organelles of the endosomal-lysosomal system during its short existence. This phenomenon occurs in all the cell types examined to date independent of whether they are professional or non-professional phagocytic cells. The formation of the PV involves the induction of a calcium flux into the host cell via the action of a parasite-derived calcium agonist, which is generated through the action of a parasitic oligopeptidase (Caler etal., 1998), as well as the synaptotagmin VII pathway (Caler et al., 2001), the recruitment of lysosomes (Tardieux et al., 1992), and the participation of microtubules (Tyler et al., 2005) and actin filaments (Rosestolato et al., 2002). Recently, Fernandes et al. (2011) showed that $T$ cruzi trypomastigotes mimic the process of wound repair with $\mathrm{Ca}^{2+}$-dependent exocytosis of lysosomes by delivering acid sphingomyelinase to the host plasma membrane, facilitating parasite entry into host cells. These aspects of the invasion process have been extensively reviewed in recent years (Hall, 1993; Burleigh and Andrews, 1995; Yoshida, 2006; Alves and Colli, 2007; de Souza et al., 2010; Caradonna and Burleigh, 2011; Butler and Tyler, 2012; Fernandes and Andrews, 2012; Romano et al., 2012).

\section{ENDOCYTOSIS IS A COMPLEX BIOLOGICAL PROCESS}

Our present knowledge of the endocytic process shows that it is more complex than previously thought. Indeed, in addition to the classical phagocytic process, there are several ways a cell can ingest extracellular material of variable dimensions. These mechanisms can be either dependent on dynamin, such as the clathrinand caveolin-mediated processes, or independent of dynamin, as occurs during processes including macropinocytosis, and lipid 
raft-mediated endocytosis. It is likely that other mechanisms will be described in view of the large number of groups attempting to better characterize the endocytic process. As described in another review in this volume (Barrias et al., submitted), T. cruzi may use all of these mechanisms to enter host cells. It is possible that the parasite selects the mechanism to be used based on factors such as the nature of the cell and the host cell surface ligand to which

\section{REFERENCES}

Alves, M. J., and Colli, W. (2007). Trypanosoma cruzi: adhesion to the host cell and intracellular survival. IUBMB Life 59, 274-279.

Andrews, N. W., Abrams, C. K., Slatin, S. L., and Griffiths, G. (1990). A T. cruzi-secreted protein immunologically related to the complement component C9: evidence for membrane pore-forming activity at low pH. Cell 61, 1277-1287.

Burleigh, B. A., and Andrews, N. W. (1995). The mechanisms of Trypanosoma cruzi invasion of mammalian cells. Annu. Rev. Microbiol. 49, 175-209.

Butler, C. E., and Tyler, K. (2012). Membrane traffic and synaptic crosstalk during host cell entry by Trypanosoma cruzi. Cell. Microbiol. 14, 1345-1353.

Caler, E. V., Vaena de Avalos, S., Haynes, P. A., Andrews, N. W., and Burleigh, B. A. (1998). Oligopeptidase B-dependent signaling mediates host cell invasion by Trypanosoma cruzi. EMBO J. 17, 4975-4986.

Caler, E. V., Chakrabarti, S., Fowler, K. T., Rao, S., and Andrews, N. W. (2001). The Exocytosis-regulatory protein synaptotagmin VII mediates cell invasion by Trypanosoma cruzi. J. Exp. Med. 193, 1097-1104.

Caradonna, K. L., and Burleigh, B. A. (2011). Mechanisms of host cell invasion by Trypanosoma cruzi. Adv. Parasitol. 76, 33-61.

Carvalho, T. M., and de Souza, W. (1989). Early events related with the behaviour of Trypanosoma cruzi within an endocytic vacuole in mouse peritoneal macrophages. Cell Struct. Funct. 14, 383-392.

de Souza, W., de Carvalho, T. M., and Barrias, E. S. (2010). Review on Trypanosoma cruzi: host cell interaction. Int. J. Cell Biol. 2010, pii: 295394.
Dvorak, J. A., and Hyde, T. P. (1973). Trypanosoma cruzi: interaction with vertebrate cells in vitro. I. Individual interactions and the cellular and subcellular levels. Exp. Parasitol. 34, 268-283.

Dvorak, J. A., Miller, L. H., Whitehouse, W. C., and Shiroishi, T. (1975). Invasion of erythrocytes by malaria merozoites. Science 187 , 748-750.

Fernandes, M. C., Cortez, M., Flannery, Andrews, N. W. (2011). Trypanosoma cruzi subverts the sphingomyelinasemediated plasma membrane repair pathway for cell invasion. J. Exp. Med. 208, 909-921.

Fernandes, M. C., and Andrews, N W. (2012). Host cell invasion by Trypanosoma cruzi: a unique strategy that promotes persistence. FEMS Microbiol. Rev. 36, 734-747.

Jones, T. C., and Hirsch, J. G. (1972). The interaction between Toxoplasma gondii and mammalian cells. II. The absence of lysosomal fusion with phagocytic vacuoles containing living parasites. J. Exp. Med. 136, 1173 1194.

Hall, B. F. (1993). Trypanosoma cruzi: mechanisms for entry into host cells. Semin. Cell Biol. 4, 323-333.

Hyde, T. P., and Dvorak, J. A. (1973). Trypanosoma cruzi: interaction with vertebrate cells in vitro. II. Quantitative analysis of the penetration phase. Exp. Parasitol. 34, 284-294.

Kofoid, C. A., Wood, F. C., and McNeil, E. (1935). The cycle of Trypanosoma cruzi in tissue cultures of embryonic heart muscle. Univ. Calif. Publ. Zool. 41, 23-24.

Kipnis, T. L., Calich, V. L. G., and da Silva, W. D. (1979). Active entry of bloodstream forms of Trypanosoma cruzi into macrophages. Parasitology 78, 89-98. A. R., Tam, C., Mortara, R. A., and binds. Once the parasite binds to and is recognized by the host cell surface it triggers a process that is better described as an induced endocytosis.

\section{ACKNOWLEDGMENTS}

The authors wish to thank the support of $\mathrm{CNPq}$, CAPES, and FAPERJ.

Langreth, S. G., and Trager, W. (1973). Fine structure of the malaria parasite Plasmodium lophurae. J. Protozool. 20, 600-613

Meyer, H., and Xavier de Oliveira, M. (1948). Cultivation of Trypanosoma cruzi in tissue culture: a four year study. Parasitology 39, 91-94.

Nery Guimarães, F., and Meyer, H. (1942). Cultivo de Toxoplasma Nicole \& Manceaux, 1909, em culturas de tecidos. Rev. Bras. Biol. 2, 123-129.

Nogueira, N., and Cohn, Z. (1976). Trypanosoma cruzi: mechanism of entry and intracellular fate in mammalian cells. J. Exp. Med. 143, 1402-1420.

Romaña, C., and Meyer, H. (1942). Estudo do ciclo evolutivo do Schizotrypanum cruzi em cultura de tecidos de embrião de galinha. Mem. Inst. Oswaldo Cruz 37, 19-27.

Romano, P. S., Cueto, J. A., Cassassa, A. F., Vanrell, M. C., Gotlieb, R. A., and Colombo, M. I. (2012). Molecular and cellular mechanisms involved in the Trypanosoma cruzi host cell interplay. IUBMB Life 64, 387-396.

Rosestolato, C. T., Dutra Jda, M., de Souza, W., and de Carvalho, T. M. (2002). Participation of host cell actin filaments during interaction of trypomastigote forms of Trypanosoma cruzi with host cells. Cell Struct. Funct. 27, 91-98.

Schenkman, S., Robbins, E. S., and Nussenzweig, V. (1991). Attachment of Trypanosoma cruzi to mammalian cells requires parasite energy, and invasion can be independent of the target cell cytoskeleton. Infect. Immun. 59, 645-654.

Tardieux, I., Webster, P., Ravesloot, J., Boron, W., Lunn, J. A., Heuser, J. E., et al. (1992). Lysosome recruitment and fusion are early events required for trypanosome invasion of mammalian cells. Cell 71, 11171130 .

Tyler, K. M., Luxton, G. W. G., Appewhite, D. A., Murphy, S. C., and Engman, D. (2005). Responsive microtubule dynamics promote cell invasion by Trypanosoma cruzi. Cell. Microbiol. 7, 1579-1591.

Yoshida, N. (2006). Molecular basis of mammalian cells invasion by Trypanosoma cruzi. Ann. Acad. Bras. Cienc. 78, 87-111.

Xu, Y., and Weiss, L. M. (2005). The microsporidian polar tube: highly specialized invasion organelle. Int. J. Parasitol. 35, 941-953.

Conflict of Interest Statement: The authors declare that the research was conducted in the absence of any commercial or financial relationships that could be construed as a potential conflict of interest.

Received: 27 August 2012; accepted: 02 January 2013; published online: 25 January 2013.

Citation: de Souza W and Ulisses de Carvalho TM (2013) Active penetration of Trypanosoma cruzi into host cells: historical considerations and current concepts. Front. Immun. 4:2. doi: 10.3389/fimmu. 2013.00002

This article was submitted to Frontiers in Microbial Immunology, a specialty of Frontiers in Immunology.

Copyright (c) 2013 de Souza and Ulisses de Carvalho. This is an open-access article distributed under the terms of the Creative Commons Attribution License, which permits use, distribution and reproduction in other forums, provided the original authors and source are credited and subject to any copyright notices concerning any third-party graphics etc. 